\title{
WAVE TRANSMISSION AT LOW-CRESTED STRUCTURES USING NEURAL NETWORKS
}

\author{
R.P. van Oosten ${ }^{1}$, J. Peixó Marco², J.W. van der Meer $^{3}$, \\ M.R.A. van Gent ${ }^{4}$, H.J.Verhagen ${ }^{5}$
}

\begin{abstract}
The European Union funded project DELOS was focused on wave transmission and an extensive database on low-crested rubble mound structures was generated. During DELOS, new empirical wave transmission formulae were derived. These formulae still showed a considerable scatter due to a limited number of parameters included. Neural networks based on a homogeneous database have resulted in a useful prediction model for wave overtopping within the EU project CLASH. The successful methodology of CLASH is applied within this study. The aim of this study is to improve the prediction of wave transmission in comparison to the empirical DELOS formulae with help of a prediction model based on neural networks. This paper gives a overview of the contents of the composed homogeneous database and gives insight in the capacity and accuracy of the final prediction model. The final prediction model includes 9 input parameters, which is more than at present in the existing hand-derived empirical formulae. The prediction model is accurate in predicting wave transmission for both smooth and mound lowcrested structures.
\end{abstract}

\section{INTRODUCTION}

Wave transmission is often an important criterion in the design of a breakwater structure and influences early decisions on the type of structure and the applied construction material. In design stages classical formulae are used to predict the wave transmission coefficient $K_{t}$. The EU funded project DELOS was focused on wave transmission and an extensive database on low-crested rubble mound structures was generated. During DELOS, new empirical wave transmission formulae were derived (Van der Meer et al., 2004), but still show a considerable scatter.

Another recently finished EU-project named CLASH was concentrating on wave overtopping. The use of a homogeneous database as a valuable source of information and using it for neural networks in combination with resampling

\footnotetext{
${ }^{1}$ Former MSc. student TU Delft, Ingenieursbureau BCC, PO Box 5094, 2600 GB Delft, The Netherlands, r.vanoosten@ingbcc.nl

${ }^{2}$ Former MSc. student, Universitat Politècnica de Catalunya, C. Jordi Girona 31, 08034 Barcelona, Spain, herzstahl@gmail.com

${ }^{3}$ Infram, PO Box 16, 8316 ZG Marknesse, The Netherlands, jentsje.vandermeer@infram.nl

${ }^{4}$ WL | Delft Hydraulics, PO Box 177, 2600 MH Delft, The Netherlands, marcel.vangent@wldelft.nl

${ }^{5}$ Delft University of Technology, Hydraulic Engineering Section, PO Box 5048, 2600 GA Delft, The Netherlands, h.j.verhagen@tudelft.nl
} 
techniques as a prediction model for wave overtopping have been shown during the CLASH project (Van der Meer et al., 2005).

The goal of the study described herein is to obtain a widely applicable prediction model for wave transmission behind various types of low-crested structures. Accuracy and simplicity are important factors that make a prediction model useful or not. Until now, wave transmission could only be predicted with classical formulae or with help of a simple neural network, which is only suitable for mound type of breakwater structures (Panizzo et al., 2005). This study is mainly focusing on improving the prediction of wave transmission with use of neural networks as a prediction model by:

- Making the prediction of the wave transmission coefficient Kt more accurate and reliable by using a homogeneous database and neural networks in combination with resampling techniques (CLASH methodology).

- Making it possible to handle both mound and smooth low-crested structures within one model, so the prediction model can be used in a wide range (for instance permeable and impermeable structures or rough and smooth structures).

When using neural networks it is possible to involve more input parameters and take into account complex relations in the prediction of the wave transmission coefficient than possible with a hand-derived empirical formula. To be able to derive these relations a sufficient amount of data is required. Also the data should represent a variety of types of structures. In this way the prediction model will be able to take into account more physics and will therefore be able to make an accurate prediction of the wave transmission coefficient.

\section{THE HOMOGENEOUS DATABASE}

As a neural network requires a huge amount of available wave transmission data, one of the main tasks of the present study was the set up of an extensive homogeneous database consisting of wave transmission test results. The homogeneous database is used to train the neural network and has therefore a direct influence on the prediction of the wave transmission coefficient. It is important and necessary to have homogeneous data in order to obtain accurate and reliable results from the prediction model. Additionally, it can be stated that the more test results the database contains, the more input-output pairs there are available for a neural network to train with. In general the results will be more accurate and the range of use will be larger. In relation to the reasons as mentioned above, a considerable part of the study was spent to compose an extensive homogeneous database. 


\section{Composure of the database}

The composed database consists of a variety of tests. Different types of structures were tested at different test facilities, under different circumstances and with use of different measure techniques. Making data homogeneous, means in principal that collected datasets are screened carefully for reliability. After this the data is added to the database in such a way, that all datasets can be described with a fixed limited number of parameters. The used parameters can be divided into: general, hydraulic and structural parameters. To have insight in these parameters, reports about the individual tests were collected and examined in detail.

For each test, imposed hydraulic and structural parameters have been determined. The database consists of 19 structural parameters and 10 hydraulic parameters, describing the available tests. Missing hydraulic parameters in front of the structure are calculated with the numerical wave model SWAN (Booij et al., 1999) and the empirical model of Battjes and Groenendijk (2000). Depending on the reliability of the measurements a 'reliability-factor' is assigned, ranging from 1 (very reliable) to 4 (unreliable). Test reports have been examined in order to judge the reliability. Also a 'complexity-factor' is assigned, depending on the difficulty of describing / characterizing the considered test section with the structural parameters, ranging from 1 (simple section) to 4 (very complex section). An overview of the most governing parameters that are present in de homogeneous database are shown in figure 1. The reliability factor and the complexity factor are combined together to a weight factor for each individual test. This weight factor implies the number of times a certain test was used to train the neural network with. In this way reliable tests with a simple cross-section are used several times more as input than less reliable tests with a difficult cross-section.

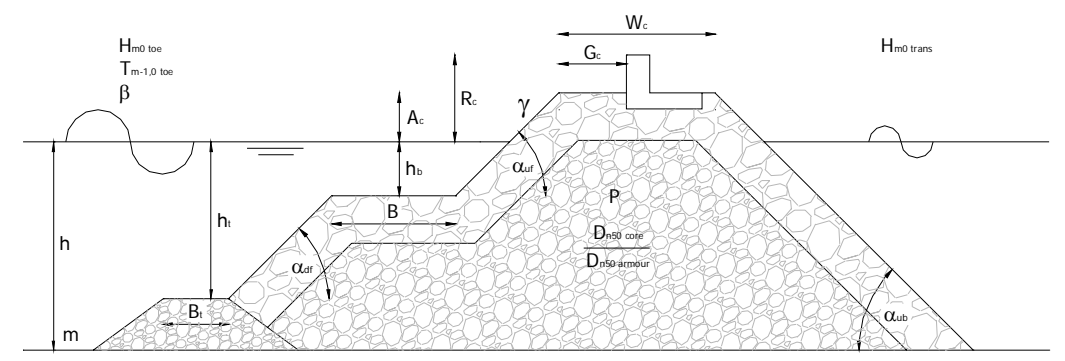

Figure 1. Governing parameters present in homogeneous database

\section{Analysis of the database}

A major part of the available data was already collected during DELOS. The DELOS database includes 2,337 wave transmission tests and is made homogeneous according to the CLASH-work database during this study. 
Another 1,597 tests have been collected and added to the database to a total number of 3,934 tests. The database consists of a variety of tested breakwater structures: mound structures (both permeable and impermeable) as well as smooth structures (impermeable). All these structures have their own characteristics and behave different for wave transmission. An important issue is to use parameters in the database that represent the characteristics of those structures. This was found possible with the major part of parameters used in the CLASH-database (Steendam et al., 2004 and Verhaeghe et al., 2003).

\begin{tabular}{|l|l|}
\hline \multicolumn{2}{|l|}{ Table 1. Types of structures in database } \\
\hline Structure type & Number of tests \\
\hline All tests & 3,934 \\
Rubble stone (rock) & 1,687 \\
Concrete elements & 367 \\
Artificial reef elements & 1,063 \\
Smooth structures & 808 \\
Composite structures & 9 \\
\hline
\end{tabular}

The distribution of data points is important to evaluate the performance of a neural network. In regions with many data points the neural network is able to find more relations and this results in a better accuracy in these regions. An analysis on the distribution of data in the homogeneous database is made in order to obtain the following information:

- Determining regions where data points are concentrated. In these regions the neural network is most accurate and is used to validate the model.

- Detecting white spots. White spots are regions where no data points are present. In those regions the neural network interpolates results and the prediction model performance was analyzed carefully in these regions. Special attention is paid to evaluate if tendencies the model is showing are physically right.

- Detecting parameters which are such likely distributed that the neural network would find no extra relations to learn with (too less variation). These parameters can be discarded before the prediction model will be trained.

The distribution of wave steepness, relative crest freeboard and relative crest width have a large influence on the prediction model. The analysis of the wave steepness and relative crest freeboard are included in this paper. 


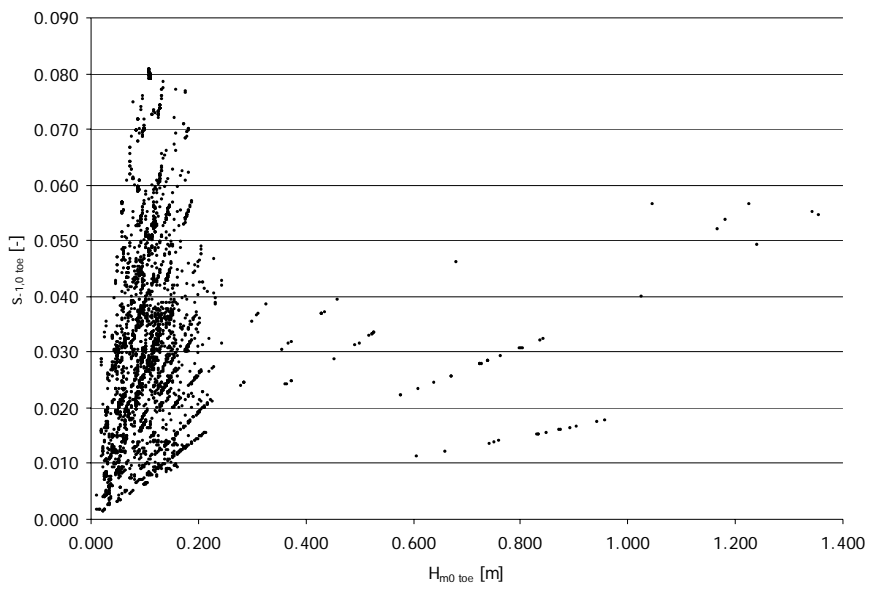

Figure 2. Distribution of wave height at toe vs. wave steepness

No prototype measurements are present in the database. The database consists of small- and large scale tests as shown in Figure 2. The figure shows the wave steepness as a function of the wave height for all available tests. A wave steepness over 0.07 is physically not possible, as the waves should break beyond this point. A wave steepness lower than 0.005 is difficult to generate in a wave flume or wave basin. Very small waves, lower than $0.03 \mathrm{~m}$ are difficult to generate as well. These data will be considered as less reliable and therefore a reliability factor of at least 3 ('less reliable test') is assigned to those individual tests.

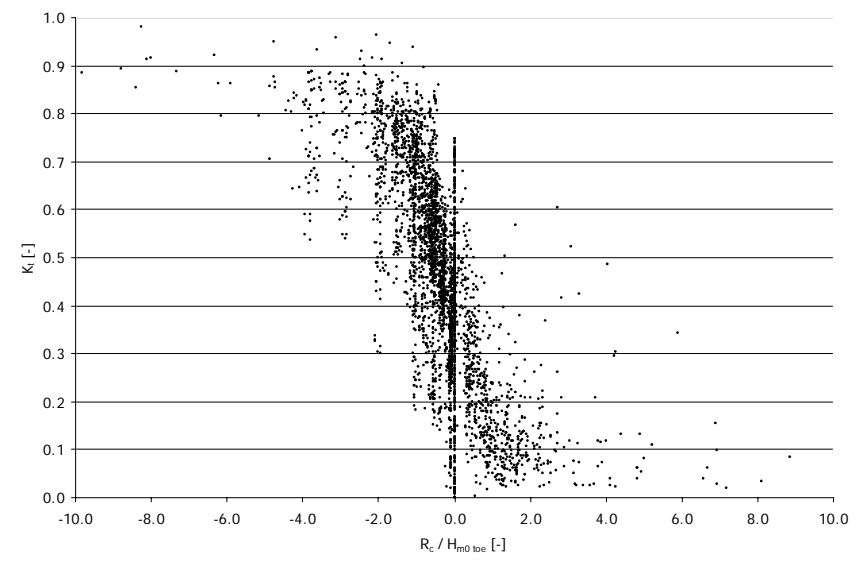

Figure 3. Distribution of relative crest height versus wave transmission coefficient 
From Figure 3 can be concluded that the concentration of data points is between relative crest freeboards of -4.0 till 4.0. Beyond those boundaries, the distribution of data points is sparse and the accuracy of the prediction model is expected to be considerably lower. There are no data points present with a $\mathrm{K}_{\mathrm{t}}=0$ or a $K_{t}=1$. Hence, the neural network will be less accurate in areas close to these values of $\mathrm{K}_{\mathrm{t}}$.

\section{Data and input parameters used in final prediction model}

The empirical formulae of DELOS (Briganti et al., 2003 and Van der Meer et al., 2004) contain a limited number of parameters. All input parameters of these empirical formulae were carefully selected and known to be of influence on wave transmission. However, due to the limited number of input parameters the empirical formulae still show a considerable scatter (see figure 2). As stated before, neural networks have the capacity to take into account more input parameters and therefore a better prediction can be obtained. However, it is very important to limit the number of input parameters to the ones which are known to influence wave transmission in order to avoid the neural network starts learning false relations. On the other hand it can be stated too that by reducing the total number of input parameters the neural network will be less complex and will therefore perform better.

The final parameters are selected, based on physical reasoning and from findings of earlier studies. The parameters that are selected for the final prediction model are presented for a typical cross-section in figure 5.

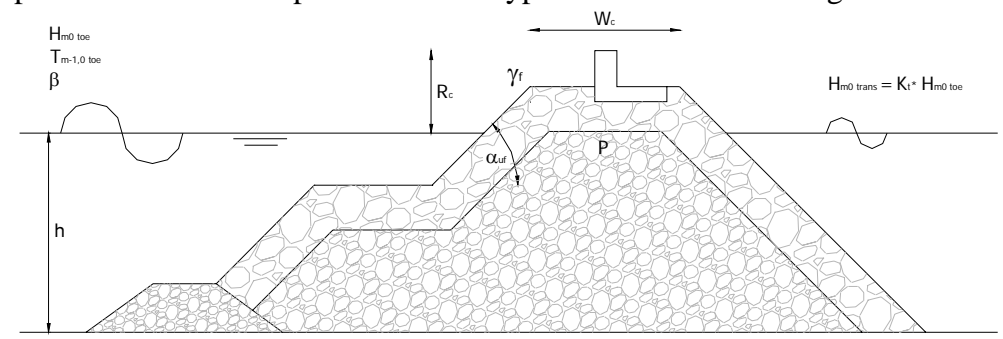

Figure 5. Overview of input parameters used for the prediction model

In comparison with the empirical formulae from DELOS new parameters are introduced to the prediction of wave transmission:

- The water depth in front of the structure h [m] is introduced in order to take into account the effect of shallow water on incident waves.

- The roughness factor $\gamma_{f}[-]$ (TAW, 2002) is introduced to distinguish rough from smooth structures.

- $\quad$ The notional permeability factor P [-] (Van der Meer, 1988a) is introduced to distinguish permeable from impermeable structures 


\section{PREDICTION MODEL}

\section{Configuration of the neural network}

The CLASH prediction method (Van Gent et al., 2004) is applied in this study as well. This is a Multilayer Perceptron Neural Network, with the Bayesian Regularization as training algorithm. This study was focused on a three-layered neural network, were a configuration of one single hidden layer was chosen. The input layer consists of 8 input parameters (figure 5). The incident wave height is used to apply Froude scaling and making the other input parameters dimensionless. An optimum of 11 neurons in the hidden layer was found in order to avoid overfitting. The output layer consists of one output parameter: the wave transmission coefficient $\mathrm{K}_{\mathrm{t}}$. The transfer function between the input layer and hidden layer is a log-sigmoid function. The transfer function between the hidden layer and output layer is a saturated linear function. The advantage of using a saturated linear function is that the output parameter only can adapt values between 0 and 1 like the physical boundaries of $\mathrm{K}_{\mathrm{t}}$.

\section{Ensemble of neural networks}

The final prediction model of this study is also carried out by means of an ensemble of neural networks. This ensemble is obtained from 100 neural networks, which are trained based on a bootstrap resampling technique (Van den Boogaard et al., 2000). The training and testing process are repeated many times to solve the problem of representativeness of the training and testing set. With the ensemble of neural networks the final prediction of the wave transmission coefficient is calculated with the mean of L predictions:

$\bar{K}_{t}=\frac{\sum_{i=1}^{L} K_{t i}}{L}$ (1)

The ensemble of NN's results in a set of predictions from which an uncertainty assessment can be made for individual input parameters. Confidence intervals give insight in the reliability of the prediction. As a result, the neural network does not give a prediction of wave transmission but also a measure for reliability of the prediction.

\section{Accuracy of the ensemble of neural networks}

Figure 6 shows that the prediction of the ensemble of neural networks is very accurate along the whole range of values of $\mathrm{K}_{\mathrm{t}}$, although some points (most of them belong to the new dataset) seem to be a bit far from the correlation line. Despite this more than $80 \%$ of the tests have an error below 0.05 (absolute value). 

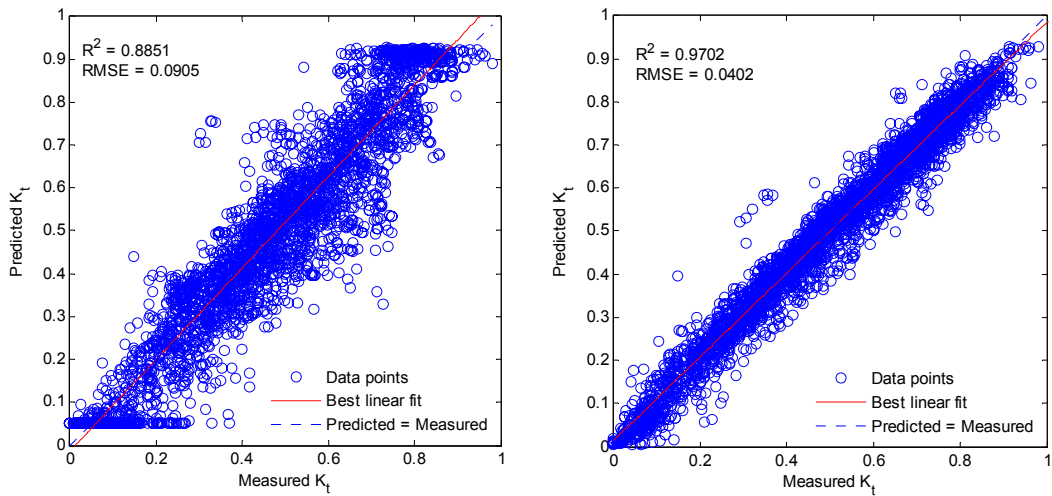

Figure 6. Comparison of prediction results DELOS (left) and the ensemble of neural networks (right)

The comparison with the empirical formulae of DELOS shows that the ensemble of neural networks results in a definitely higher accuracy. The main reason is that more parameters were used than for the hand-derived empirical approach. Of course this is one of the advantages of a neural network. The improvements when using the ensemble of neural networks are:

- High accuracy (the lowest $\mathrm{R}^{2}$ is 0.9262 for smooth structures and the highest $\mathrm{R}^{2}$ is 0.9715 for mound structures).

- No important over- or under prediction and more than the $80 \%$ of the predicted $K_{t}$ have a deviation below 0.05 from the measured $K_{t}$ in the overall prediction.

- $\quad$ No lower and upper limits on $\mathrm{K}_{\mathrm{t}}$ other than the physical limits 0 and 1.

\section{VALIDATION AND RELIABILITY OF THE PREDICTION MODEL}

As mentioned before, in total 9 input parameters are found to be most optimal for the prediction model for wave transmission. All parameters relevant for a reliable prediction of the wave transmission coefficient are taken into account, representing different behaviors for various types of breakwater structures. All parameters that are used in the DELOS formulae and in the present prediction model too are showing physically tendencies between the given boundaries. The parameters that were introduced new for the prediction of wave transmission have shown to be important. 
The final input boundary of individual input parameters is based on:

- $\quad$ Physical boundary. For every parameter there is a physical range present within a certain parameter may vary. Values outside this range are physically impossible and can logically not be entered to the prediction model.

- Data distribution. The data distribution on which the model is based, determines the range where the model is expected to give a reliable prediction. In regions with a lot of data points the model will give reliable predictions, contrary to regions with few or no data points. The boundaries are based on the $2.5 \%$-quantile and $97.5 \%$-quantile data distribution, representing the $95 \%$ data interval of the total amount of available data points, because the neural network is able to interpolate within that range.

- Model validation. With help of sensitivity analysis of individual parameters, the provided tendencies are validated with earlier findings from different studies on wave transmission. An upper and lower boundary of regions is determined where the model predicts physical right tendencies.

\section{Boundaries of input parameters}

Within the boundaries of input parameters as shown in table 2 and table 3 prediction model gives reliable predictions of the wave transmission coefficient. All three boundaries have resulted in the normative final input boundaries of the prediction model. Within the given ranges the model is capable of providing reliable predictions. Different boundaries are determined for mound and smooth structures as these structures behave very differently to wave transmission.

\begin{tabular}{|l|l|}
\hline \multicolumn{2}{|l|}{ Table 2. Prediction model input boundaries for smooth structures } \\
\hline Input Parameter & Input boundary \\
\hline Incident significant wave height, $\mathrm{H}_{\mathrm{m} 0 \text { toe }}[-]$ & $\begin{array}{l}\mathrm{H}_{\mathrm{m} 0 \text { toe }}>0.03 \mathrm{~m} \\
\mathrm{H}_{\mathrm{m} 0 \text { toe }} / \mathrm{h}<0.50\end{array}$ \\
\hline Mean wave steepness, $\mathrm{s}_{0 \mathrm{~m}-1,0 \text { toe }}[-]$ & $0.009<\mathrm{S}_{0 \mathrm{~m}-1,0}<0.060$ \\
\hline Angle of wave incidence, $\beta\left[{ }^{\circ}\right]$ & $0^{\circ}<\beta<70^{\circ}$ \\
\hline Relative water depth, $\mathrm{h} / \mathrm{H}_{\mathrm{m} 0 \text { toe }}[-]$ & $1.20<\mathrm{h} / \mathrm{H}_{\mathrm{m} 0 \text { toe }}<9.80$ \\
\hline Relative crest freeboard, $\mathrm{R}_{\mathrm{c}} / \mathrm{H}_{\mathrm{m} 0 \text { toe }}[-]$ & $-2.00<\mathrm{R}_{\mathrm{c}} / \mathrm{H}_{\mathrm{m} 0 \text { toe }}<0.70$ \\
\hline Relative crest width, $\mathrm{W}_{\mathrm{c}} / \mathrm{H}_{\mathrm{m} 0 \text { toe }}[-]$ & $0.60<\mathrm{W}_{\mathrm{c}} / \mathrm{H}_{\mathrm{m} 0 \text { toe }}<6.00$ \\
\hline Front slope, cot $\alpha_{\mathrm{uf}}[-]$ & $1.0<\cot \alpha_{\mathrm{uf}}<3.8$ \\
\hline Roughness factor, $\gamma_{\mathrm{f}}[-]$ & 1.0 \\
\hline Notional Permeability factor, $\mathrm{P}[-]$ & 0.10 \\
\hline
\end{tabular}




\begin{tabular}{|l|l|}
\hline \multicolumn{2}{|l|}{ Table 3. Prediction model input boundaries for mound structures } \\
\hline Input Parameter & Input boundary \\
\hline Incident significant wave height, $\mathrm{H}_{\mathrm{m} 0 \text { toe }}[-]$ & $\begin{array}{l}\mathrm{H}_{\mathrm{m} 0 \text { toe }}>0.03 \mathrm{~m} \\
\mathrm{H}_{\mathrm{m0}} / \mathrm{h}<0.50\end{array}$ \\
\hline Mean wave steepness, $\mathrm{S}_{0 \mathrm{~m}-1,0 \text { toe }}[-]$ & $0.006<\mathrm{S}_{0 \mathrm{~m}-1,0}<0.080$ \\
\hline Angle of wave incidence, $\beta\left[^{\circ}\right]$ & $\begin{array}{l}0^{\circ}<\beta<45^{\circ}, \text { but enter as } \\
\text { input value: } \beta=0^{\circ}\end{array}$ \\
\hline Relative water depth, $\mathrm{h} / \mathrm{H}_{\mathrm{m} 0 \text { toe }}[-]$ & $1.55<\mathrm{h} / \mathrm{H}_{\mathrm{m} 0 \text { toe }}<11.00$ \\
\hline Relative crest freeboard, $\mathrm{R}_{\mathrm{c}} / \mathrm{H}_{\mathrm{m} 0 \text { toe }}[-]$ & $-3.50<\mathrm{R}_{\mathrm{c}} / \mathrm{H}_{\mathrm{mo} \text { toe }}<1.80$ \\
\hline Relative crest width, $\mathrm{W}_{\mathrm{c}} / \mathrm{H}_{\mathrm{m} 0 \text { toe }}[-]$ & $0.01<\mathrm{W}_{\mathrm{c}} / \mathrm{H}_{\mathrm{m} 0 \text { toe }}<47.00$ \\
\hline Front slope, cot $\alpha_{\mathrm{u}}[-]$ & $1.0<\cot \alpha_{\mathrm{uf}}<5.0$ \\
\hline Roughness factor, $\gamma_{\mathrm{f}}[-]$ & $0.38<\gamma_{\mathrm{f}}<0.90$ \\
\hline Notional Permeability factor, $\mathrm{P}[-]$ & $0.10<\mathrm{P}<0.60$ \\
\hline
\end{tabular}

\section{Model validation}

A model validation can be performed by means of a sensitivity analysis. Figures 7, 8 and 9 are examples of a sensitivity analysis.

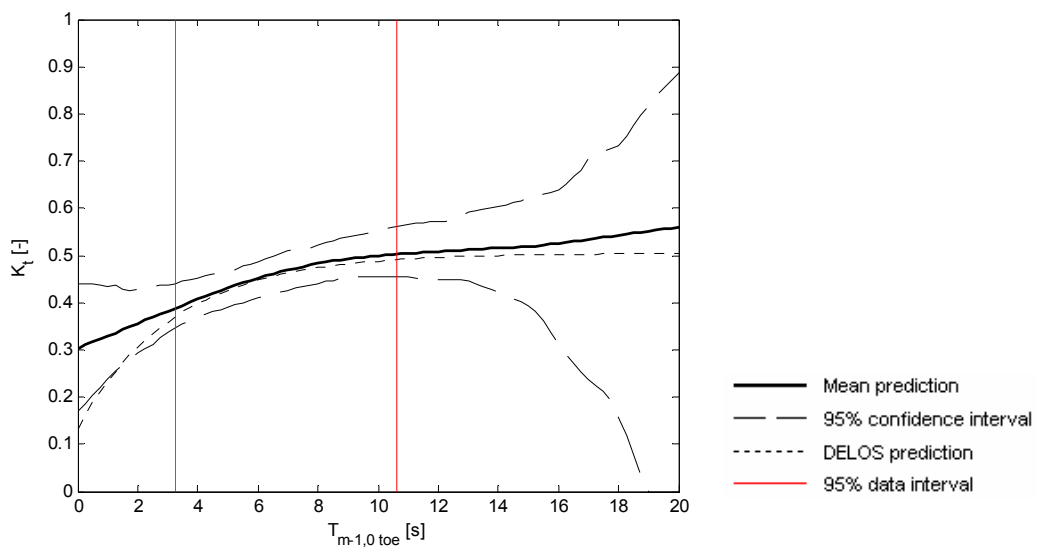

Figure 7. Example 1: Sensitivity of $T_{m-1,0}$ toe for a submerged mound structure

The first example concerns wave transmission for a submerged mound structure. The influence of the mean wave period is clear and physically right. A longer wave passes a structure more easily, resulting in a higher transmission coefficient. The prediction model shows the expected tendency for both submerged, emerged, smooth and mound structures. The confidence interval is narrow between the data boundaries, indicating the prediction model is unambiguous in predicting the wave transmission coefficient. Note that the result of the prediction model is very close to the result of the empirical formula of DELOS. 


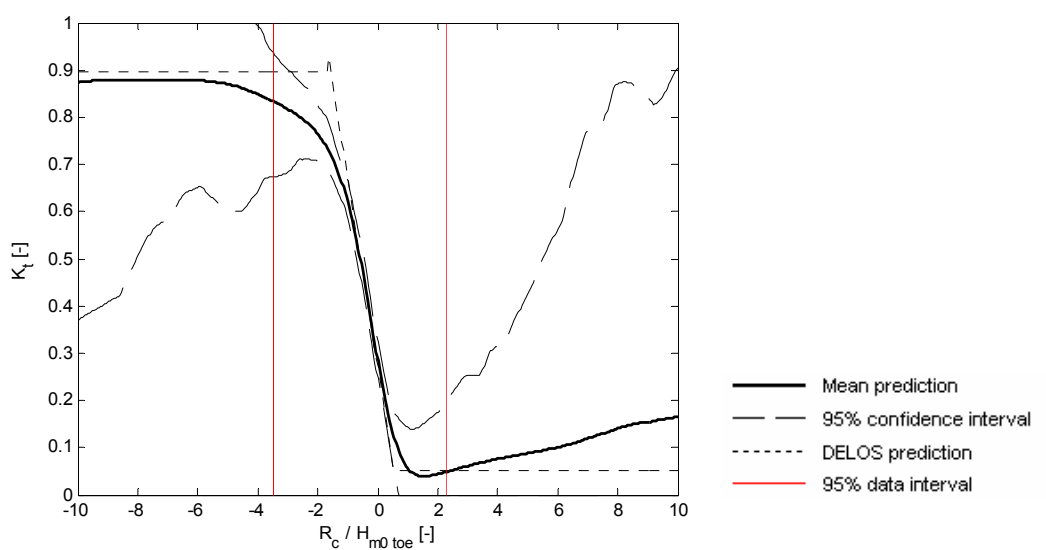

Figure 8. Example 2: Sensitivity of $R_{c} / H_{m 0}$ toe for a mound structure

The influence of the relative crest freeboard in Example 2 (figure 8) is very clear. An increasing relative crest freeboard results in a decreasing transmission coefficient, which is physically expected. Within the range of the test data (95\% confidence interval) this tendency is very strong and the confidence band is very narrow. It is expected that an increasing relative crest freeboard always results in a decreasing transmission coefficient. Within the range of $-8.0<\mathrm{R}_{\mathrm{c}} / \mathrm{H}_{\mathrm{m} 0 \text { toe }}<$ 1.8 this tendency is shown by the prediction model. For $\mathrm{R}_{\mathrm{c}} / \mathrm{H}_{\mathrm{m} 0 \text { toe }}>1.8$ the model shows a physically wrong prediction, which is caused by the limited number of data points within this region (see figure 3).

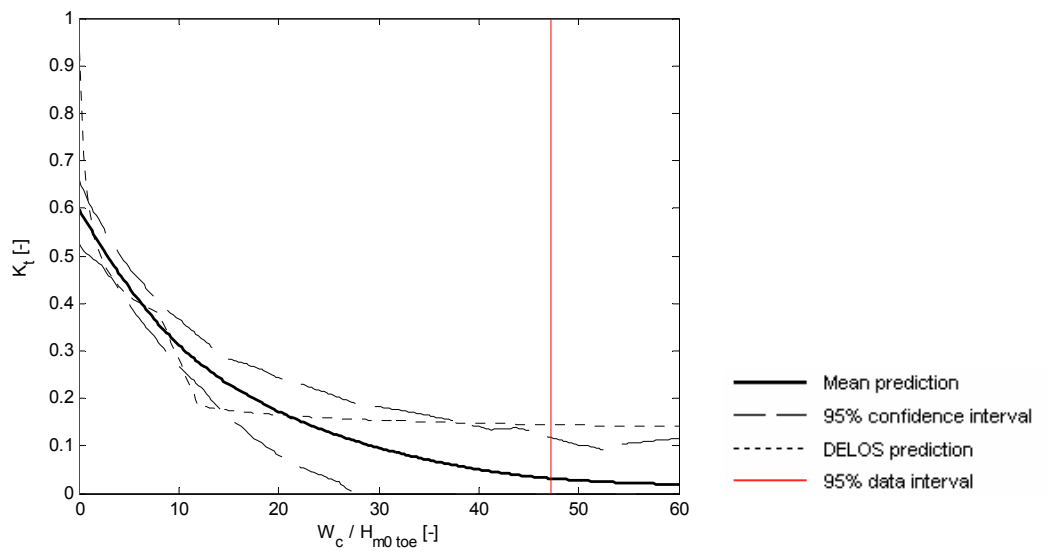

Figure 9. Example 3: Sensitivity of $\mathrm{W}_{\mathrm{c}} / \mathrm{H}_{\mathrm{mo}}$ toe for a submerged mound structure 
Example 3 (figure 9) concerns wave transmission for a submerged mound structure. The prediction model shows a decreasing transmission coefficient for an increasing relative crest width for a submerged mound structures, which is physically right. This tendency continues till a constant transmission coefficient is reached. Note that the transmission coefficient is decreasing to zero, which is to be expected as a rough crest keeps causing wave energy dissipation as a wave travels over the crest. The range of validity of the prediction model to this relative crest width is equal to the boundary of the data distribution.

For smooth structures only a slight decreasing transmission coefficient is found as the relative crest width increases (no figure included). This is completely deviating from mound structures. This indicates that the prediction model clearly separates the behavior of smooth structures from mound structures concerning the influence of the relative crest width. Additionally, the influence of the relative crest width is much smaller for smooth structures compared to mound structures. This difference is explained in earlier studies by the fact that much less energy dissipation is present at the crest of a smooth structure, contrary to mound structures where energy dissipation is present due to friction and permeability effects of the rough and permeable crest.

\section{CONCLUSIONS}

A homogeneous database on wave transmission is composed with a total number of 3,934 wave transmission test results. The database has been used for the training of a neural network prediction model for wave transmission. The prediction model is able to improve the prediction of the wave transmission coefficient compared to existing empirical formulae in terms of accuracy and reliability.

By introducing the roughness factor and the notional permeability factor it is found possible to handle both mound and smooth structures within one prediction model. The prediction model is able to distinguish permeable from impermeable structures and rough from smooth structures as these kinds of structures are known to behave completely different for wave transmission.

By using an ensemble of neural networks it is possible to perform an uncertainty assessment with the prediction model. Therefore, besides predicting a wave transmission coefficient, information is provided about the reliability of the predicted transmission coefficient. An uncertainty assessment is not possible with existing hand-derived empirical formulae for wave transmission. 


\section{ACKNOWLEDGEMENTS}

The authors would like to thank Prof. Francisco Taveira Pinto (Universidade do Porto), Dr. Karl-Friedrich Daemrich (Universität Hannover) and WL | Delft Hydraulics for making their datasets available.

\section{REFERENCES}

Battjes, J.A. and H.W. Groenendijk, 2000. "Wave height distributions on shallow foreshores”. Journal of Coastal Engineering, No. 40, 161-182.

Booij, N., Ris, R.C., Holthuijsen, L.H. (1999). "A third generation wave model for coastal regions, Part I, Model description and validation, Journal of Geophysical Research, 104, C4, pp. 7649-7666.

Briganti R, Van der Meer J.W., Buccino M., Calabrese M. (2003). "Wave transmission behind low crested structures”, Proc. $3^{\text {rd }}$ Coastal Structures Conference.

Panizzo, A., Briganti, R., Van der Meer, J.W., Franco, L., (2003). “Analysis of wave transmission behind low crested structures using neural networks", Proc. $4^{\text {th }}$ Int. Coastal Structures Conference 2003, Portland, Oregon.

Steendam, G. J., van der Meer, J. W., Verhaeghe, H., Besley, P., Franco, L. and M. van Gent, 2004. "The international database on wave overtopping”, ASCE, Proc. ICCE 2004, Lisbon

TAW (2002). “Technisch rapport golfoploop en golfoverslag bij dijken” (Technical report on wave runup and wave overtopping at dikes - in Dutch). Technical Advisory Committee on Water Defences, The Netherlands

Van den Boogaard, H., Mynett, A.E. and Heskes, T. (2000), "Resampling techniques for the assessment of uncertainties in parameters and predictions of calibrated models", Proc. Hydroinformatics Conference.

Van Gent, M.R.A., van der Boogaard, H.F.P. (1998). "Neural network modelling of forces on vertical structures", Proc. $26^{\text {th }}$ Int. Conf. on Coastal Engineering, ASCE, pp. 2096-2109.

Van der Meer, J.W. (1988a). "Rock slopes and gravel beaches under wave attack”, Phd-Thesis, Delft University of Technology. Also: Delft Hydraulics Communication No. 396

Van der Meer, J.W., Briganti, R., Wang, B. and Zanuttigh, B. (2004), "Wave transmission at low-crested structures including oblique wave attack”, Proc. $29^{\text {th }}$ Int. Conf. on Coastal Engineering, ASCE.

Van der Meer, J.W., M.R.A. van Gent, B. Pozueta, H. Verhaeghe, G.J. Steendam and J.R. Medina, 2004. "Applications of a neural network to predict wave overtopping at coastal structures”. ICE, Proc. Coastlines, Structures and Breakwaters 2005, London.

Verhaeghe, H., Van der Meer, J.W., Steendam, G.-J., Besley, P., Franco L. And Van Gent, M.R.A. (2003). "Wave overtopping database as the starting point for a neural network prediction method” ASCE, Proc. Coastal Structures 2003, Portland 